\title{
ON THE RADICAL OF THE GROUP ALGEBRA OF A p-NILPOTENT GROUP
}

\author{
R. J. CLARKE \\ (Received 26 September 1969) \\ Communicated by G. E. Wall
}

\section{Introduction}

In this note we give a basis for the radical of the group algebra of a $p$-nilpotent group over a field of characteristic $p$ in terms of the ordinary representation theory of the group. We use our result to calculate the exponent of the radical for such a group.

Notation. Let $p$ be a fixed prime, $k$ an algebraically closed field of characteristic $p$ and $G$ a finite group. Denote by $k G$ the group algebra of $G$ over $k$ and by $N=$ $N(G)$ the radical of $k G$. We denote the radical of a general finite dimensional $k$-algebra $A$ by $\operatorname{rad} A$. Let $G$ have order $|G|$. We assume throughout that $p$ divides $|G|$, in which case $N \neq 0$. By a $k G$ module we mean a left $k G$ module.

\section{Lemmas}

We begin with two results which are perhaps of independent interest.

Lemma 1. Let $H$ be a normal $p^{\prime}$-subgroup of $G$ and $L$ an irreducible $k H$ module. Write $E=\operatorname{End}_{k G}\left(L^{G}\right), F=\operatorname{rad} E$ and $N=N(G)$. Then, using the natural (right) action of $F$ on $L^{G}$,

$$
N^{i} \cdot L^{G}=L^{G} \cdot F^{i} \text { for all } i \geqq 1 .
$$

Proof. We may take $L=k H e$ for some primitive $k H$ idempotent $e$, and $L^{G}=k G e$. Write $1=e_{1}+e_{2}+\cdots+e_{n}$, a sum of primitive $k H$ idempotents, with $e=e_{1}$.

$$
\begin{aligned}
N \cdot L^{G}=N e & =k G N e \\
& =k G e N e+k G e_{2} \mathrm{Ne}+\cdots+k G e_{n} N e
\end{aligned}
$$

as left $k G$ modules, where the sum is not necessarily direct.

Now $e_{i} k G e \cong \operatorname{Hom}_{k G}\left(k G e_{i}, k G e\right)$ as $k$-spaces under the map

$$
a \rightarrow \varphi \in \operatorname{Hom}_{k G}\left(k G e_{i}, k G e\right)
$$


where $b \varphi=b a$ for all $b$ in $k G e_{i}$. We use this fact to show that $N e=k G e N e$.

Let $f_{i}$ be the primitive central $k H$ idempotent corresponding to $e_{i}, 1 \leqq i \leqq n$. Denote by $N_{G}\left(f_{i}\right)$ the group of elements of $G$ commuting with $f_{i}$ and by $T_{i}$ a left transversal for $N_{G}\left(f_{i}\right)$ in $G$. Then $F_{i}=\sum_{g \in T_{i}} f_{i}^{g}$ is a central $k G$ idempotent. Now if $f_{1}$ and $f_{i}$ are not conjugate in $G, F_{i} F_{1}=0$. Hence

$$
e_{i} k G e=e_{i} f_{i} F_{i} k G F_{1} f_{1} e_{1}=0 .
$$

Suppose $f_{1}$ and $f_{i}$ are conjugate in $G$, say $f_{1}=f_{i}^{g}$. Now

$$
e_{i}^{g} f_{1}=\left(e_{i} f_{i}\right)^{g}=e_{i}^{g} .
$$

Hence $e_{i}^{g}$ and $e$ are in the same $k H$ block $k H f_{i}$. Since $H$ is a $p^{\prime}$-group we may use ordinary representation theory to deduce that $k H e \cong k H e_{i}^{g}$. Thus

$$
k G e \cong k G e_{i}^{g} \cong k G e_{i}
$$

We claim that in this case $e_{i} N e=e_{i} k G e N e$. For there is an $a \in e_{i} k G e$ such that the map $\varphi: k G e_{i} \rightarrow k G e$ given by $x \varphi=x a$ is an isomorphism. Hence there is a $b \in e k G e_{i}$ such that $y \varphi^{-1}=y b$ for all $y \in k G e$. Hence $x a b=x$ for all $x$ in $k G e_{i}$. Thus

$$
e_{i}=e_{i} a b=\left(e_{i} a\right) b=a b .
$$

Let $c \in e_{i} N e$.

$$
c=e_{i} c=a(b c) \in e_{i} k G e N e .
$$

Thus $e_{i} N e \subset e_{i} k G e N e$. Since the reverse inclusion is obvious we have equality. Hence

$$
\begin{aligned}
k G e_{i} N e & =k G e_{i} k G e N e \subset k G e N e, \\
N e & =k G e N e=(k G e)(e N e) .
\end{aligned}
$$

Now by [1] 54.6 we know that $e N e$ and $F$ are identical as rings. Hence $N \cdot L^{G}=$ $L^{G} \cdot F$. Thus our result holds for $i=1$.

Suppose $N^{j} \cdot L^{G}=L^{G} \cdot F^{j}$ for all $j \leqq i$, i.e.

$$
N^{j} e=(k G e)(e N e)^{j}
$$

Multiplying (1) on the left by $N$ gives

$$
N^{j+1} e=(N e)^{j+1},
$$

whereas multiplying (1) on the right by $\mathrm{Ne}$ gives

$$
\left(N^{j} e\right)(N e)=(k G e)(e N e)^{j+1} .
$$


Thus $N^{i+1} e=(N e)^{i+1}$, using (2) with $j=i$

$$
\begin{aligned}
& =(N e)^{i}(N e) \\
& =\left(N^{i} e\right)(N e), \text { using (2) with } j=i-1 \\
& =(k G e)(e N e)^{i+1}, \text { using (3) with } j=i .
\end{aligned}
$$

Therefore $N^{i+1} \cdot L^{G}=L^{G} \cdot F^{i+1}$. The result follows by induction.

Defintion. If $H$ is normal in $G$ and $L$ is a $k H$ module, the stabilizer $S=S(L)$ of $L$ in $G$ is defined by

$$
S=\left\{g \in G ; L^{g} \cong L\right\} .
$$

LEMMA 2. In the situation of Lemma 1 , if $S$ is the stabilizer of $L$ in $G, N^{i} \cdot L^{G}=$ $k G \cdot N(S)^{i} \cdot L^{S}$ for all $i \geqq 1$.

Proof. Let $g_{1}, \cdots, g_{s}$ be a left transversal for $H$ in $S$ and $g_{1}, \cdots, g_{n}$ a left transversal for $H$ in $G$.

$$
\begin{aligned}
& L^{S}=\bigoplus_{i=1}^{s} g_{i} \otimes L \text { is a } k S \text { submodule of } \\
& L^{G}=\bigoplus_{i=1}^{n} g_{i} \otimes L \text { and } L \text { is a } k H \text { submodule of } L^{S} .
\end{aligned}
$$
where

Let $\theta \in \operatorname{End}_{k S}\left(L^{S}\right)$ and define $\varphi: \operatorname{End}_{k S}\left(L^{S}\right) \rightarrow \operatorname{End}_{k G}\left(L^{G}\right)$ by putting $\varphi(\theta)=\theta^{\prime}$,

$$
\left(g_{i} \otimes l\right) \theta^{\prime}=g_{i}(l \theta), l \in L, i=1, \cdots, n,
$$

and extending $\theta^{\prime}$ linearly to $L^{G}$. It is well known and easy to prove that $\varphi$ is an isomorphism of rings such that $\theta$ and $\varphi(\theta)$ have the same action on $L$.

Thus

$$
\begin{aligned}
& N^{i} \cdot L^{G}=\left(\sum_{j=1}^{n} g_{j} \otimes L\right)\left(\operatorname{rad} \operatorname{End}_{k \mathrm{G}}\left(L^{G}\right)\right)^{i} \text {, by Lemma } 1, \\
& =\sum_{1}^{n} g_{j}\left(L \cdot\left(\operatorname{rad} \operatorname{End}_{k G}\left(L^{G}\right)\right)^{i}\right) \\
& =\sum_{1}^{n} g_{j}\left(L \cdot\left(\operatorname{rad} \operatorname{End}_{k S}\left(L^{S}\right)\right)^{i}\right) \\
& \subset k G \cdot N(S)^{i} L^{S} \text {, by Lemma } 1 .
\end{aligned}
$$

The reverse inclusion is proven similarly. Hence the result follows.

\section{3. p-nilpotent groups}

Let $G$ be a $p$-nilpotent group with Sylow $p$-subgroup $P$ and normal $p$-complement $H$. Let $e$ be a primitive idempotent of $k H$ and put $L=k H e$. Suppose $L$ has stabilizer $S=H Q$ in $G$, where $Q$ is a Sylow $p$-subgroup of $S$. Now 


$$
E=\operatorname{End}_{k S}\left(L^{S}\right) \cong \underset{q \in Q}{\bigoplus} \operatorname{Hom}_{k H}(L, q \otimes L) .
$$

We know from [3] that there is a unique $k S$ module $X$ such that $\left.X\right|_{H}=L$. Let $X$ afford the representation $\rho$ of $S$ with respect to the $k$-basis $W$.

For each $q \in Q$ the map $T_{q}: L \rightarrow q \otimes L$ given by

$$
l T_{q}=q \otimes \rho\left(q^{-1}\right) l, l \in L,
$$

is a $k H$-isomorphism.

Therefore $\left\{T_{q} ; q \in Q\right\}$ is a $k$-basis for the right hand side of (4). $E$ therefore has $k$-basis $\left\{\eta_{q} ; q \in Q\right\}$, where $\eta_{q}$ is defined by

$$
\begin{aligned}
\left(q^{\prime} \otimes l\right) \eta_{q} & =q^{\prime}\left(l T_{q}\right) \\
& =q^{\prime} q \otimes \rho\left(q^{-1}\right) l, q^{\prime} \in Q, l \in L .
\end{aligned}
$$

Now $\eta_{q^{\prime}} \eta_{q}=\eta_{q^{\prime} q}$. Hence $E \cong k Q$. Thus rad $E$ has basis $\left\{\eta_{1}-\eta_{q} ; q \in Q-\{1\}\right\}$ Define $\eta(q, l)=1 \otimes l-q \otimes \rho\left(q^{-1}\right) l, q \in Q, l \in L$.

THEOREM 1. The set $\{\eta(q, l) ; q \in Q-\{1\}, l \in W\}$ is a $k$-basis for $N(S) L^{S}$.

Proof. $N(S) L^{S}=L^{S} \cdot \operatorname{rad} E$. Now

$$
\begin{aligned}
\left(q^{\prime} \otimes l\right)\left(\eta_{1}-\eta_{q}\right) & =q^{\prime} \otimes l-q^{\prime} q \otimes \rho\left(q^{-1}\right) l \\
& =-\eta\left(q^{\prime}, \rho\left(q^{\prime}\right) l\right)+\eta\left(q^{\prime} q, \rho\left(q^{\prime}\right) l\right), \text { and } \\
(1 \otimes l)\left(\eta_{1}-\eta_{q}\right) & =\eta(q, l) .
\end{aligned}
$$

Hence the result follows.

We can now give an explicit expression for $N(G)$. For let $1=e_{1}+\cdots+e_{n}$ be a decomposition of $1 \in k H$ into primitive orthogonal idempotents. Write $L_{i}=$ $k H e_{i}$ and let $L_{i}$ have stabilizer $S_{i}$ in $G$. Let $S_{i}$ have Sylow $p$-subgroup $Q_{i}$. Then

$$
\begin{aligned}
k G & =\oplus k G e_{i}=\oplus L_{i}^{G} \text { as left } k G \text { modules and } \\
N & =\sum N \cdot L_{i}^{G} \\
& =\sum k G \cdot N(S) \cdot L_{i}^{S_{i}} \text {, which can be calculated. }
\end{aligned}
$$

Definition. The exponent of $N(G)$ is the least integer $n$ such that $N(G)^{n}=0$.

THEOREM 2. If $G$ is p-nilpotent and $P$ is a Sylow p-subgroup of $G$ then $N(G)$ and $N(P)$ have the same exponent.

Proof. We use the previous notation.

Consider the idempotent $f=\sum_{h \in H} h /|H|$ of $k G$. It is easy to show that $k G f \cong k P$ as algebras. Hence

$$
\begin{aligned}
N(G)^{n}=0 & \Rightarrow(\operatorname{rad}(k G f))^{n}=0 \\
& \Rightarrow(\operatorname{rad} k P)^{n}=0 \\
& \Rightarrow N(P)^{n}=0 .
\end{aligned}
$$


Conversely, let $N(P)^{n}=0$. We have that

$$
\begin{aligned}
N(G)^{n} & =\sum_{i} N(G)^{n} L_{i}^{G} \\
& =\sum_{i} k G \cdot N\left(S_{i}\right)^{n} L_{i}^{S_{i}} \text { by Lemma } 2, \\
& =\sum_{i} k G \cdot L_{i}^{S_{i}}\left\{\operatorname{rad}_{\left.\operatorname{End}_{k S_{i}}\left(L_{i}^{S_{i}}\right)\right\}^{n} \text { by Lemma } 1 .}\right.
\end{aligned}
$$

Now $Q_{i}$ is contained in some Sylow $p$-subgroup $P_{1}$ of $G$, so

Since

$$
N\left(Q_{i}\right)^{n} \subset N\left(P_{1}\right)^{n}=0 .
$$

we have

$$
\operatorname{End}_{k S_{i}}\left(L_{i}^{S_{i}}\right) \cong k Q_{i}
$$

Therefore $N(G)^{n}=0$.

$$
\left\{\operatorname{rad} \operatorname{End}_{k S_{i}}\left(L_{i}^{S_{i}}\right)\right\}^{n}=0 .
$$

REMARKS. If $G$ is a group of $p$-length one then $G$ contains a normal $p$-nilpotent subgroup $K$ such that $G / K$ is a $p^{\prime}$-group. By results of Highman [2] and Villamayor [4] we have that $N(G)=k G \cdot N(K)$. Theorem 2 therefore holds for groups of $p$-length one. Similar calculations can be carried out in the case of a general $p$-soluble group. However Theorem 2 does not hold in such a case. The exponent of $N(G)$ may be greater than or less than that of $N(P)$.

The material in this paper was part of my thesis submitted for the degree of Doctor of Philosophy at the University of Warwick. I should like to express my gratitude to Professor J. A. Green for his help and encouragement during the preparation of this work.

I gratefully acknowledge the support of a British Council Commonwealth Scholarship.

\section{References}

[1] C. W. Curtis and I. Reiner, Representation Theory of Finite Groups and Associative Algebras (New York 1962).

[2] D. G. Higman, 'On modules with a group of operators', Duke Math. J. 21 (1954), 369-376.

[3] B. Srinivasan, 'On the indecomposable representations of a certain class of groups', Proc. London Math. Soc. 10 (1960), 497-513.

[4] O. E. Villamayor, 'On the semisimplicity of group algebras II', Proc. Amer. Math. Soc. 10 (1959), 27-31.

Mathematics Department

University of Adelaide 